\title{
Living Experiences of Indian Adult Cancer Survivors - a Brief Report
}

\author{
Bidhu Kalyan Mohanti ${ }^{1}$, Jaspreet Kaur ${ }^{2 *}$
}

\begin{abstract}
Background: This pilot study looked into the physical, social, psychological and economic issues of Indian adult cancer survivors. Materials and Methods: Assumed cancer free patients, after cancer directed therapy (CDT), were assessed on the basis of a questionnaire developed by the investigators. The mental status of the survivors was elicited by modified MINI international neuro-psychiatric interview. This cross sectional assessment was conducted as a direct interaction with each patient for 30 to 45 minutes at a cancer hospital in 2012. Results: Thirty one adult cancer survivors participated in this study. Median age was 53 years with a median follow up duration of 21.8 months (Range 2.3-194.1 months). The majority $(68 \%)$ did not receive financial support for treatment. Median interval after CDT to start of activity of daily living was 1.5 months (range: 0-24 months). Fatigue and loss of appetite were reported by $52 \%$ and $29 \%$ respectively. The cancer diagnosis and its treatment adversely impacted the financial condition in $42 \%$ of patients. Nineteen percent each showed social anxiety and post-traumatic stress disorder and another $13 \%$ patients reported depression. Conclusions: This prospective assessment highlights survivorship issues and the need to address those issues particularly in the context of developing countries where resources and manpower are limited.
\end{abstract}

Keywords: Adult cancer survivorship - questionnaire - outcomes - physical - psychosocial issues - India

Asian Pac J Cancer Prev, 16 (2), 507-512

\section{Introduction}

It is estimated that in 2006, there were 25 million persons alive with cancer worldwide and the survivors will increase to approximately 75 million by 2030 (Boyle, 2006; Siegel et al., 2012). Among the great medical success stories of the $20^{\text {th }}$ century was our ability to turn cancer, once almost uniformly fatal, into an illness that is curable for more people, and could be controlled in most. Contributing factors to this success in the last 50 years are namely, cancer screening and early detection, effective combined treatments, and better supportive care. As a result, the population of long-term cancer survivors accounts for approximately $30 \%$ and $65 \%$ of all cancer patients in developing countries and USA respectively (Pandey and Thomas, 2001; Siegel et al., 2012). "A cancer survivor is living with and beyond cancer". Family members, friends, and caregivers are also affected by the survivorship experience and are also included in this definition. However, the present cancer care models largely focus on treatment aspects, cure, disease failure and survival rates and most oncology specialists have little formal training in the survivorship care plan (Office of Cancer survivorship, 2005). In recent years, the field of cancer survivorship research has been developed to help us better understand the human and social challenges of living long term after cancer diagnosis (Ganz, 2007; Siegel et al., 2012). It is now recognized that cancer survivors are at increased risk of developing various physical and psychosocial conditions, which need to be identified and attended within the cancer care system (Nord et al., 2007). There are four main areas of assessment which can affect the daily living and quality of life of a cancer survivor: physical (symptoms), functional (capacity to engage in activities of daily living), emotional (mood/affective and cognitive), and social (role functioning and/or need for support, financial burden).

During the last decade or so, the most significant studies have been done on childhood cancer survivors in USA, Europe, Australia and other developed countries (Leisenring et al., 2009). There is a growing awareness in adult oncology that a distinct phase comes in the cancer trajectory where the treatment is completed and the patient transitions into a period of less intense medical supervision. The Institute of Medicine, USA in 2006 made certain key recommendations for the adult cancer survivorship, namely, 1 .routine provision of a treatment summary, 2.a survivorship plan to be explained to the patient (and family) at the end of active cancer therapy, 3.psychosocial, nutrition and other supportive services

${ }^{1}$ Department of Radiation Oncology, Fortis Memorial Research Institute, Sector, Gurgaon, ${ }^{2}$ Department of Radiation Oncology, Dr.B.R.A. Institute Rotary Cancer Hospital (IRCH), All India Institute of Medical Sciences(AIIMS), Ansari Nagar, New Delhi, India *For correspondence: drjaspreet.ro@gmail.com, 
to be provided as part of long-term care (Hewitt et al., 2006). A recent study from the cancer survivors in USA has revealed that reliable information related to their health status should be provided by trusted sources like the treating physicians (Shahrokniet et al., 2014).

It is observed that cancer care models in the developing countries are yet to recognize the needs of cancer survivors (Pandey and Thomas, 2001). Till the last decade, the cancer cure rates were largely low and the oncologists were not encountering a sizeable number of cancer survivors in India. At present many cancer centers have patients who are on follow-up for months to years after diagnosis and treatment. At this center (i.e. Dr BRA. Institute Rotary Cancer Hospital (IRCH), All India Institute of Medical Sciences (AIIMS), New Delhi) approximately 60,000 cancer patients are on follow-up visits every year (AIIMS, 52nd Annual Report, 2007-08, www.aiims.edu.). Out of these, nearly $30 \%$ are long-term survivors.

We report a prospective pilot study on Indian adult cancer survivors, identifying the various survivorship issues. A proforma was designed (cancer survivorship questionnaire(CSQ)) to document the patient's disease and treatment details and obtain subjective responses from the patients related to the physical, functional, social, economic and mental aspects of daily living. In order to assess the mental status of the survivors, we have also included the widely used and internationally accepted MINI (Mini International Neuropsychiatric Interview version, www.psychtoolkit.com/mini-screen-600.html) along with the CSQ in this survey (Sheehan et al., 1999).

\section{Materials and Methods}

This study evaluated the cancer patients visiting Dr BRAIRCH AIIMS after a cancer diagnosis and completion of curative cancer directed therapy (CDT). This study was approved by the Institutional ethics committee (IEC/NP-252/2010) and is registered at the protocol registration system of Clinical Trials.gov as ID number NCT01304199.

\section{Aims and objectives}

The aims and objectives of this study were 1 . to examine and identify sequelae of cancer and its treatment. 2. to study the various physical, psychological, social and economic issues which the cancer survivors face. Patients aged 18 years or above, of both sexes who received CDT and, remained disease free for at least 3 months after cancer diagnosis and therapy were entered into this study. Patients were explained about this cancer survivorship assessment and those who agreed to give consent were recruited into the study. Patients who received anti-cancer treatment outside the institute or had recurrence or disease failure at the time of assessment were excluded.

\section{Cancer survivorship assessment:}

Subjects were evaluated and assessed for their cancer survivorship issues on the basis of a questionnaire (CSQ) developed by the investigators after the literature review. This CSQ consisted of 45 questions; divided into three parts: to capture subjective responses related to 1. Patients' personal details, 2. Cancer diagnosis and treatment details, 3. Survivorship status survey-mainly regarding physical, social, and financial issues. The mental status of the survivors was assessed by the MINI, the assessment tool utilized internationally by the clinicians and researchers and is freely allowed for research and public institution purposes. Cancer diagnosis and post therapy the psychological morbidities are an important component of cancer survivorship. MINI is able to elicit the features of post traumatic stress disorder (PTSD) such as anxiety, depression and adjustment disorder. This module was modified for the socio-cultural context of its application to cancer survivors in India.

\section{Survey methodology and study type}

The survey was conducted at the Dr BRA Institute Rotary Cancer Hospital (IRCH), AIIMS over the calendar year of 2012. The patient and family caregiver were given due privacy and time of around 30-45 minutes for the doctor-patient interaction during the survey process.

This is a cross sectional study of empirical nature, conducted as a onetime point evaluation within the hospital premise. It was a direct face to face interaction between one member of the treating physician's team and patient (with or without family caregivers). In case of relevant missing information, the same patient or family were contacted subsequently for completeness of the survey. The analysis was carried out to determine socioeconomic characteristics, impact of cancer diagnosis and CDT, physical and psychological survivorship issues post- therapy. The adverse physical and psychological experiences were calculated as proportion out of the total accrued patients.

\section{Results}

A pilot study was conducted on 31 adult cancer survivors in whom the median age was 53 years (range 26-70 years) and male to female ratio was 3.4:1. Head and neck cancer $(48.4 . \%)$ was the most common cancer followed by gastrointestinal malignancies $(35.5 \%)$ in this small study population. Fifteen patients $(48.4 \%)$ had advanced stage and $10(32.2 \%)$ were of early stage at the time of presentation, with 6 in unknown stage. Median survivorship duration, in these 31 patients, was 21.8 months (range 3.3-194.1 months). About 84\% (26/31) patients had undergone more than one anti-cancer treatment.

\section{Socio-economic characteristics (Table 1)}

Approximately 50\% (16/31) of the patients had education till school level. Median income of survivors was Indian Rupees (INR) 15,500 (range 1000-55000 INR; median USD $=290$ ) per month. Most of the patients were self employed (42\%), 7 did not have any earning. Median amount of money spent during anti-cancer treatment was 75000 INR (range: 6000-500,000; median USD=1390). Sixty eight percent of patients experienced loss of their income, median amount of loss was 31000 INR, while on treatment. A similar proportion of patients did not receive any financial support for their CDT. 
Table 1. Patient Characteristics

\begin{tabular}{|c|c|c|c|}
\hline Median Age & \multicolumn{2}{|c|}{$\begin{array}{c}53 \text { Years } \\
\text { (Range:26-70 Years) }\end{array}$} & $\begin{array}{l}\text { Percentage } \\
\quad(\%)\end{array}$ \\
\hline \multirow[t]{2}{*}{ Sex } & Male & 24 & 77 \\
\hline & Female & 7 & 23 \\
\hline \multirow[t]{3}{*}{ Education } & School level & 16 & 52 \\
\hline & Graduate or above & 11 & 35 \\
\hline & No education & 4 & 13 \\
\hline \multirow[t]{3}{*}{ Occupation } & Self employed & 19 & 61 \\
\hline & Gov. employed & 5 & 16 \\
\hline & No earning & 7 & 23 \\
\hline \multirow[t]{4}{*}{ Site of Cancer } & Head and Neck & 15 & 49 \\
\hline & Gastrointestinal & 11 & 35 \\
\hline & Gynecological & 2 & 7 \\
\hline & Others & 3 & 9 \\
\hline \multirow[t]{3}{*}{ Cancer Stage } & Stage I+II & 10 & 32 \\
\hline & Stage III +IV & 15 & 49 \\
\hline & Not staged & 6 & 19 \\
\hline \multirow[t]{4}{*}{ Cancer Therapy } & Surgery & 15 & 49 \\
\hline & Chemotherapy & 16 & 52 \\
\hline & Radiotherapy & 27 & 87 \\
\hline & More than 1 cancer treatment & 26 & 84 \\
\hline Money spent during treatment (Median and range in Indian Rupees) & \multicolumn{3}{|c|}{ Rs $75000(6000-5$ lakh $)$} \\
\hline Loss of Income during treatment (Number of patients) & & 21 & 68 \\
\hline Loss of Income during treatment (Median and range) & \multicolumn{3}{|c|}{ Rs $31,000(10,000-400,000)$} \\
\hline \multirow[t]{2}{*}{ Support for Cancer treatment } & Yes & 10 & 32 \\
\hline & No & 21 & 78 \\
\hline
\end{tabular}

Total Number of Patients: 31; Median Follow up Duration: 21.8 Months (2.3-194.1 months)

Table 2. Cancer Diagnosis and Treatment Records

\begin{tabular}{|c|c|c|c|}
\hline & & Months & $(\%)$ \\
\hline \multirow[t]{2}{*}{ Information about Cancer Diagnosis } & By Doctors & 29 & 93 \\
\hline & By Relatives & 2 & 7 \\
\hline \multirow[t]{3}{*}{ Explanation of Treatment at the start of Cancer Therapy } & By Doctors & 26 & 84 \\
\hline & By Relatives & 1 & 3 \\
\hline & Learned as the Patient Received treatment & 4 & 13 \\
\hline \multicolumn{3}{|c|}{ Start of Treatment as per Expectation of Patient and Family (Yes in Numbers) } & $90 \%$ \\
\hline & Surgery & $12-15$ & \\
\hline & Chemotherapy & $15 / 16$ & \\
\hline & Radiotherapy & $26 / 27$ & \\
\hline \multirow[t]{4}{*}{ Help in Bringing the patient to Hospital } & Family Caregiver & 19 & 61 \\
\hline & Other relative & 8 & 30 \\
\hline & Others & 3 & 9 \\
\hline & Treatment Morbidity: Grade 3/4 & 16 & 52 \\
\hline Start of ADL after cancer treatment (Median and range, in Months) & 1.5 Months $(0-24 \mathrm{Mo}$ & onths) & \\
\hline \multirow[t]{8}{*}{ Most Bothersome daily activity } & Pain & 7 & 23 \\
\hline & Speech difficulty & 7 & 23 \\
\hline & Swallowing difficulty & 6 & 19 \\
\hline & Sleep disruption & 6 & 19 \\
\hline & Memory & 4 & 13 \\
\hline & Hearing difficulty & 3 & 9 \\
\hline & Irregular Bowel habits & 3 & 9 \\
\hline & More than 1 bothersome daily activity & 12 & 39 \\
\hline
\end{tabular}

Cancer Diagnosis, treatment and its sequelae (Table 2)

Information about cancer diagnosis and explanation about CDT course was provided by the treating physician to $93 \%$ and $84 \%$ respectively. Median interval after completion of anti-cancer treatment to start of activity of daily living was 1.5 months (range: 0-24 months). Thirty nine percent patients had more than one difficult symptom burden. More than $90 \%$ patients started the cancer treatment in time. Sixteen patients $(52 \%)$ experienced grade $3 / 4$ bothersome treatment-related morbidities. The follow-up schedule was explained to $93 \%$ of patients by the treating physician at completion of cancer therapy.

Physical and Social Survivorship issues (Table 3)

Physical activity stated as 'not as healthy as before' or 'very poor' was observed in $61 \%$ patients. Tiredness (fatigue) was reported by $52 \%$ and some loss of appetite by $29 \%$ of patients. Sleep disturbance in $16 \%$ and fear of cancer recurrence in $35 \%$ were observed. A change in the faith as regards religion and God was reported by $45 \%$ 


\section{Table 3. Suvivorship Issues Identified from this Survey (CSQ-Cancer Survivorship Questionnaire)}

\begin{tabular}{|c|c|c|c|}
\hline \multirow[t]{3}{*}{ Physical activity as compared to before treatment } & Same as before & 12 & $39 \%$ \\
\hline & Not as healthy as before & 17 & $54 \%$ \\
\hline & Very poor & 2 & $7 \%$ \\
\hline \multirow[t]{3}{*}{ Feeling Tiredness and need to rest ${ }^{`}$} & Not at all & 13 & $49 \%$ \\
\hline & A little & 11 & $35 \%$ \\
\hline & Quite a bit & 5 & $16 \%$ \\
\hline \multirow[t]{3}{*}{ Appetite after cancer treatment } & Good appetite eat well & 20 & $70 \%$ \\
\hline & Good appetite, not able to eat well & 7 & $23 \%$ \\
\hline & Poor appetite, not able to eat well & 2 & $7 \%$ \\
\hline \multicolumn{4}{|c|}{ Effect of cancer diagnosis \& its treatment on financial condition $(25 / 31)$} \\
\hline & Same as before & 12 & $39 \%$ \\
\hline & Affected slightly & 10 & $32 \%$ \\
\hline & Affected very much & 3 & $9 \%$ \\
\hline \multirow[t]{3}{*}{ Worry about own cancer(26/31) } & Not at all & 15 & $49 \%$ \\
\hline & A little & 9 & $29 \%$ \\
\hline & Quite a bit & 2 & $7 \%$ \\
\hline \multirow[t]{3}{*}{ Sleep } & Not disturbed at all & 24 & $77 \%$ \\
\hline & Slightly disturbed & 4 & $13 \%$ \\
\hline & Disturbed on some nights & 1 & $3 \%$ \\
\hline \multirow[t]{3}{*}{ Change in faith in religion and God after cancer treatment } & Remains same & 15 & $49 \%$ \\
\hline & More religious, God fearing & 11 & $35 \%$ \\
\hline & Do not think about religion/ less religious now & 3 & $9 \%$ \\
\hline \multirow[t]{5}{*}{ Advice regarding lifestyle modification after cancer therapy } & Smoking & 16 & $52 \%$ \\
\hline & Alcohol & 9 & $29 \%$ \\
\hline & Weight reduction & 1 & $3 \%$ \\
\hline & Daily exercise & 9 & $29 \%$ \\
\hline & Dietary modification & 8 & $26 \%$ \\
\hline Knowledge of Follow up Tests (Number of Patients) & & 26 & $84 \%$ \\
\hline
\end{tabular}

\section{Table 4. Psychological Issues}

\begin{tabular}{lcc}
\hline Psychological status & $\begin{array}{c}\text { Yes } \\
\text { (number of patients) }\end{array}$ & $\begin{array}{c}\text { Percentage } \\
(\%)\end{array}$ \\
\hline 1.Depression & & \\
-on regular basis & 4 & 13 \\
-feeling of uselessness & 4 & 13 \\
-overexcitement and irritability & 7 & 23 \\
-Urge for self abuse & 1 & 3 \\
2. Hallucination & 0 & - \\
3.Social Anxiety & & \\
-feeling anxious in public & 1 & 3 \\
-feeling uneasy in public & 3 & 9 \\
-feeling anxious/worried about routine things & 2 & 7 \\
4. Post traumatic stress disorder & 6 & 19 \\
\hline
\end{tabular}

patients. The cancer diagnosis and its treatment adversely impacted the financial condition in $42 \%$ of patients. Change in personal and social relationship after cancer diagnosis and its treatment was observed in $29 \%$ patients.

\section{Psychological issues (Table 4)}

This study identified overall depression in $52 \%$ by the modified MINI tool. The depression on a regular basis in $13 \%$ patients and 12 others (39\%) reported feeling of uselessness, overexcitement, irritability or an urge for self abuse. Social anxiety and post-traumatic stress disorder were elicited in $19 \%$ of patients.

\section{Discussion}

Most therapeutic modalities for cancer are associated with a spectrum of late morbidities ranging from minor and treatable to serious or, occasionally, potentially lethal. Long term cancer survivors report a decline in physical activity, dietary intake and overall quality of life (Blaney et al., 2013). As a result, it is increasingly important to understand the unique medical and psychosocial needs of survivors and be aware of resources that can assist patients, caregivers, and health care providers in navigating the various phases of cancer survivorship (Siegel et al., 2012).

This pilot study consisting of a small number of long term cancer survivors is the first attempt to evaluate survivorship issues from India, with a median survival of 22 months, similar to published report from Australia (Eakin et al., 2006). Patients had a median education of 10 years(not higher than school level) and this is likely to compromise their understanding about treatment course and its sequel, compliance to prescribed treatment, and coping with cancer survivorship. More than half of the patients were self employed with a limited household earning or no earning. Despite being treated in a public hospital, the median amount spent on anti-cancer treatment was approximately five times than their monthly income (INR 75000 vs 15500; USD 1390 vs 290). An earlier survey at this institute had estimated that the average monthly income was similarly one-fifth or less than the cancer therapy expenditure and this could be a reason for poor compliance (Mohanti et al., 2011), whereas in US the average treatment cost equals the monthly income (Warren et al., 2008). The financial limitations of patients from low and middle income countries contribute to the hurdles in treatment and follow-up. To add to this, majority of patients $(68 \%)$ in this study did not receive any support for their treatment, indicating a need for change in the government policy for low income cancer patients (Mohanti et al., 2011). Recent study evaluating the influence of socio-economic determinants has shown that cancer survivors with low education and low income 
status are likely to avoid proper follow up within the health system. It is imperative to pay attention to this group in their survivorship trajectory (Jung, 2014). A study from Turkey also showed that patients with higher education and younger age had more information seeking behavior (Kav, 2012)

The cancer and its treatment had an adverse impact on the financial status of $42 \%$ of our cancer survivors. For the common cancers in US, the treatment costs under managed care in 2002 ranged approximately from $\$ 21000$ to $\$ 41000$. In a 2006 US survey, almost a quarter of insured patients reported using most or all of their savings on treatment and the insurance plan paid less than expected. In this scenario, it is reported that the medical fraternity has little knowledge about the costs borne by the patients and their families (Warren et al., 2008; Elkin and Bach, 2010).

Despite the patient burden faced by major public hospitals in India, in this study, more than $90 \%$ of patients were adequately informed about the cancer diagnosis and treatment course; and similar proportion of patients experienced the start of cancer therapy well in time. More than $80 \%$ patients received multiple anti-cancer modalities and $52 \%$ experienced grade $3 / 4$ bothersome treatment morbidity. The main symptoms were fatigue, pain, loss of appetite, and insomnia. In this small cohort nearly $40 \%$ had more than one bothersome physical symptom on a daily basis. Nearly $60 \%$ stated their health as "not as healthy as before' or 'very poor'. This could have resulted in lack of motivation towards physical activity. A recent questionnaire survey of cancer survivors from UK showed that a total of $73.5 \%$ reported fatigue, with $57.2 \%$ reporting this on a regular basis, impacting adversely their quality of life (Blaney et al., 2013). Similarly, a Korean study has revealed that more than $50 \%$ of cancer survivors carry out low level of physical activity and as emphasized the need for exercise engagement in order to improve the quality of life in the survivors (Moon et al., 2013).

We observed that a median interval of 1.5 months (range: 0-24 months) was needed by the patients after completion of anti-cancer treatment to start their activity of daily living. A study about the symptom burden among US cancer survivors showed the rates of ongoing pain, psychological distress, and insomnia to be $34 \%, 26 \%$, and $30 \%$ respectively, and they were significantly higher (all $\mathrm{p}<0.001)$ than controls without a history of cancer $(18 \%, 16 \%$, and 17\%) (Mao et al., 2007). Another study from Australia showed that longer-term cancer survivors reported significant decrements in health status, days out of role, and mental well-being compared to the normal population (Eakin et al., 2006). Studies have shown that cancer survivors' physical activity levels decline by at least one third following diagnosis (Blanchard et al., 2003). A recent study on breast cancer survivors showed problems with sleep quality, stress, relationships, exercising, and maintaining body weight and cancer diagnosis increase the healthy behavior of survivors (Wang \& Chung, 2012)

The fear of cancer recurrence and a change in their faith towards God and religion were reported by $35 \%$ to $45 \%$ of patients in this study. Current literature report rates of fear of cancer recurrence, similar to our result, ranging $33-56 \%$ amongst cancer survivors and this has been shown to cause a major psychological distress (Siegel et al., 2012; Lebel et al., 2013). Approximately one third noticed a change in relationships either at personal or social levels.

In the present study, assessment of their psychological status showed different range of depression, feeling of uselessness, irritability, in more than half of the cancer survivors. Nearly $19 \%$ of patients showed both social anxiety and post-traumatic stress disorder. Thirteen percent patients reported depression on regular basis. A population survey of 863 long term cancer survivors in Australia showed that only $9 \%$ reported clinically important levels of anxiety and 4\% reported depression (Boyes et al., 2009). Although the present study involved a small number of patients, it highlights the need for routine psychological assessment and counseling of cancer survivors by the health care providers in India.

This study comprising of a small number of cancer survivors from India, at a median survival time of 22 months showed the value of systematic assessment. However the report is of limited value because of the small sample size.

In conclusion, our survey on cancer survivors in India has elicited several pertinent aspects of daily living which need the attention of healthcare system. An average patient made the out-of-pocket expenditure towards cancer therapy amounting to nearly five times of the monthly income and nearly two-thirds did not receive any financial support. Forty percent of survivors experienced more than one bothersome physical symptom. Not feeling as healthy as before, fatigue and loss of appetite were adverse experiences in $61 \%, 52 \%$, and $29 \%$ respectively. A simultaneous change in faith in religion and depressive behavior were observed in $45 \%$ and $52 \%$ respectively. This pilot study was able to identify the different issues which the cancer survivors face and there is the need to address those issues particularly in the context of developing countries where resources and manpower are limited.

\section{References}

Blanchard CM, Denniston MM, Baker F, et al (2003). Do adults change their lifestyle behaviors after a cancer diagnosis. Am J Health Behav, 27, 246-256.

Blaney JM, Lowe-Strong A, Rankin-Watt J, Campbell A, Gracey JH (2013). Cancer survivors' exercise barriers, facilitators and preferences in the context of fatigue, quality of life and physical activity participation: a questionnaire-survey. Psychooncol, 22, 186-94.

Boyes AW, Girgis A, Zucca AC, Lecathelinais C (2009). Anxiety and depression among long-term survivors of cancer in Australia: results of a population-based survey. Med J Aust, 190, 94-8.

Boyle P(2006). The globalisation of cancer. Lancet, 368, 629-30. Eakin EG, Youlden DR, Baade PD, et al (2006). Health status of long-term cancer survivors: results from an Australian population-based sample. Cancer Epidemiol Biomarkers Prev, 15, 1969-76.

Elkin EB, Bach PB (2010). Cancer's next frontier: addressing the high and increasing costs. JAMA, 303, 1086-87.

Ganz PA (2007). Cancer Survivorship. Today and tomorrow(Ed). Springer 2007. NY. ISBN-10:0-387-34349-0.

Hewitt M, Greenfield S, Stovall E (2006). From cancer patient to cancer survivors: lost in transition. Institute of Medicine, 
Washington DC, National Academic Press.

Jung M (2014). Associations of self-rated health and socioeconomic status with information seeking and avoiding behavior among post- treatment cancer patients. Asian Pac J Cancer Prev, 15, 2231-8.

Kav S, Tokdemir G, Tasdemir R, Yalili A, Dinc D (2012). Patients with cancer and their relatives beliefs, information needs and information-seeking behavior about cancer and treatment. Asian Pac J Cancer Prev, 13, 6027-32.

Lebel S, Tomei C, Feldstain A, Beattie S, McCallum M (2013). Does fear of cancer recurrence predict cancer survivors' health care use? Support Care Cancer, 21, 901-6.

Leisenring WM, Mertens AC, Armstrong GT, et al (2009). Pediatric cancer survivorship research: Experience of the childhood cancer survivor study. J Clin Oncol, 27, 2319-27.

Mao JJ, Armstrong K, Bowman MA, et al (2007). Symptom burden among cancer survivors: impact of age and comorbidity. Am Board Fam Med, 20, 434-43.

Mohanti BK, Mukhopadhyay A, Das S, Sharma K, Dash S (2011). The Economic burden of cancer. Econ Polit wkly, 43, 112-7.

Moon SH, Lee DT, Son Y (2013). Adherence to health-related lifestyle behavior recommendations and association with quality of life among cancer survivors and age-matched controls in Korea. Asian Pac J Cancer Prev, 14, 2949-54.

Nord C, Ganz PA, Aziz N, Fossa SD (2007). Follow up of long term cancer survivors in Nordic countries. Acta Oncol, 46, 433-40.

Office of Cancer Survivorship (2005). CCPS, Cancer control \& population sciences. national cancer institute.

Pandey M,Thomas BC (2001). Rehabilitation of cancer patients. $J$ Postgrad Med, 47, 62-5.

Shahrokni A, Mahmoudzadeh S, Lu BT (2014). In whom do cancer survivors trust online and offline? Asian Pac J Cancer Prev, 15, 6171-6.

Sheehan DV, Lecrubier Y, Sheehan KH, et al (1998). The Mini-International Neuropsychiatric Interview (M.I.N.I.): the development and validation of a structured diagnostic psychiatric interview for DSM-IV and ICD-10. J Clin Psychiatry, 59, 22-33.

Siegel R, DeSantis C, Virgo K, et al (2012). Cancer treatment and survivorship statistics, CA-Cancer J Clin, 62, 220-41.

Wang HH, Chung UL (2012). Healthy lifestyle changes during the period before and after cancer diagnosis among breast cancer survivors. Asian Pac J Cancer Prev, 13, 4769-72.

Warren JL, Yabroff KR, Meekins A, et al (2008). Evaluation of trends in the cost of initial cancer treatment. J Natl Cancer Inst, 100, 888-97. 